$$
\text { Cong-800804--15 }
$$

CONF-800804--45

DE83 010278

W. H. Lin

Assistant Mechanical Engineer. Compenenls Technology Oivision. Argonne National Laboratory. Argonne, IIt. $\quad 60439$

\section{Acoustic Loading Effects on Oscillating Rod Bundles}

This puper is concerned with the analyrical study of the interaction berween an infinite acoustic medium and a cluster of circular rods, The oconstic field dwe to oscillating rods and the acoustic loaling on the rods are first solved in a clased form. The acoustic loading is then used as a forcing function for rod responses, ind the acousto-elastic couplings are solved simultaneously. Numerical examples are tresented for several cases to illustrate the effects of various system parameters on the acoustic reaction force coefficients. The effect of the acoustic foading on the coupled eigenfrequencies are discussed.

\section{Introduction}

Acoustic padiation and scattering due to oscillating structures and acoustic loading on the structures are companion effects in the interaction of nuid and solid. The motion of one or more structures in the acoustic medium will cause disturbances to be radiated and scattered into the fluid as sound waves. These sound waves may again excite the structures into motion. The interaction repeats itself over and over, resulting in a coupling effect between the morion of the fluid and the structure.

In the past, analytical studies of acousto-elastic $\because:$ ation of slender rods were primarily made based on a single rod consideration [1-5]. For a group of elastic rods in a ciense acoustic medium, the motion of a rod sill excite the surrounding ones because of the fluid coupling; hence, there are many coupled modes and the rods will respond as a group rather than as a single rod [6]. The object of this paper is to provide a general methodology to solve the coupled miotion between an infinite acoustic medium and a group of circular elastic' rods.

\section{Acoustic Loading on Oscillating Rods}

Consider a group of $\boldsymbol{K}$ circular cylincters ${ }^{2}$ oscillating harmonically in an inviscid and adiabatically compressibie nuid medium at rest, as shown in Fig. 1. Assuming that the disturbances caused by the motion of the cylinders are small enough such that the linearization theory is applicable, and that the cylinders are infinitely long and their axes are parallel so that the axial wave propagntion is negligible, then the motion of the perturbed fluid is described by a twodimensional linear acoustic wave equation [7]

$$
\nabla \phi \times \frac{1}{c^{2}} \frac{\partial^{2} \phi}{\partial t^{2}}
$$

\footnotetext{
"7iercin. "elastic" is deduced from the "Bernoult:-Euterian" theory.

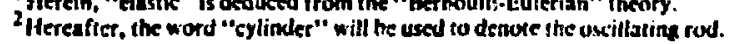

Contributed by the Pressure Vesseis and Piping Division and presenied at the Cencury 2 Pressure Vessets and Pipina Conferente, Sun Francisco. California. August 12-15. 1900. or Ter Amenucan Soctetr of MEthanical Entrimeres. Manuscript reteived al ASME Headquarlers A pril 9. $19 \mathrm{ml}$; revised manuscrip received April 24, 1911 . Paper No. 30-C2/PVP-124.

Note: ApproprialsSI Conversion Units -1 in. $=2.54 \mathrm{~cm}, 1$ fi $\cdot 0.3048 \mathrm{~m}$
}

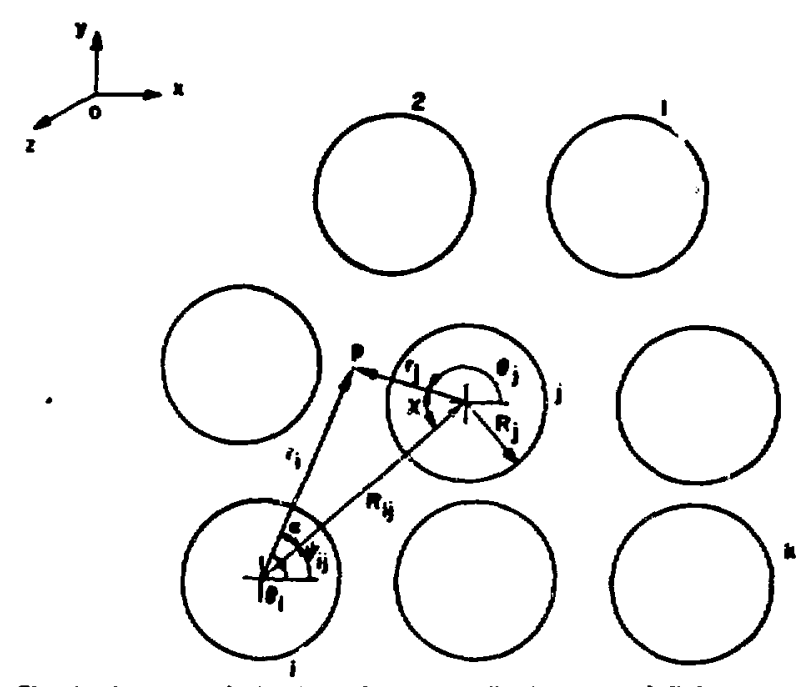

Fig. 1 a group of circular cylindars oscilluting in en intinlte com. prossible fluid

and the associaled acoustic pressurc is obtained as

$$
p=\rho_{-} \frac{\partial \boldsymbol{p}}{\partial t}
$$

where $\phi$ is the perlurbed velocity polential of the fluid, $c$ the speed of sound of the unperturiberl medium, $p_{-}$the density of the undisturbed fluid, and

$$
v^{2}=\frac{\partial^{2}}{\partial r^{2}}+\frac{1}{r} \frac{\partial}{\partial r}+\frac{1}{r^{2}} \frac{\partial^{2}}{\partial \theta^{2}}
$$

in two-dimensional cylindrical coordinates.

The appropriate boundary conditions are: 1) the normal component of the Mtidd velocity at the boundary of a cylinder is equal to the vibrational yelocity of the cylinder in that direction, and 2) the wave has to satisiy the Sommerfeld radiation condition.

The solution to equation (1) for the velocity potential of the perluribances caused by the motion of $K$ circular cylinders is composed of the partial fields due to each cylinder, anmely

\section{NOTHEE}

POATHOAS OF THIS REPORT ARE LLEAOLE. It has been reprodsced from the west avallable copy to permit the brosdest. masslble avallability.

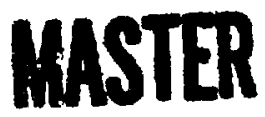

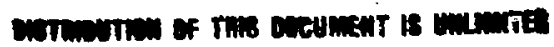




$$
\phi=\sum_{i=1}^{x}
$$

where

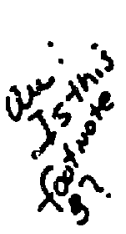

$$
\phi_{i}\left(r_{i}, \theta_{i}, t\right)=\sum_{m=0}^{\infty}\left(A_{i m} \cos m \theta_{i}+B_{i m} \sin m \theta_{i}\right)
$$

which includes all the possible radiation and scattering of the acoustic fields generated by the oscillating cylinder, numbered $i$, with other cylinders being kept stationary. $H_{m}^{\prime \prime}(k r$,$) is the$ Hankel function of the first kind of order $m$ with argument $k r_{i}$, and $\left(r_{i}, 0_{i}\right)$ are the circular coordinutes associated with cylinder $i$. When $k r$, becomes large, $H_{m}^{\prime \prime}\left(k r, e^{-1 \text { tw }}\right.$ beliaves as outgoing waves; hence, the Summer feld radiation condition is satisfied.

The total field $\Phi$ in equation (3) is written in terms of the local circular coordinates of each cylinder. To satisfy the boundary condition at the surface of a particular cylinder, the total field is expressed in ecrms of the circular coordinates of that cylinder by using the transformation of the cylindrical wave functions [8]. In doing so and using the boundary condition at each cylinder, one can reduce the problem of the acoustic radiation and stattering to an infinite set of algebraic equations for the unknown coefficicnts $A_{l m}$ and $B_{b m}$. By truncating the index $m$ to a finite number, the resulting equations can be solved for $A_{i m}$ and $B_{i m}$ by a high-speed digital computer.

Knowing $A_{1 m}$ and $B_{m m}$, the total field $\Phi$ is solved and the related quantities such as the pressure distribution, the reaction force, and the acoustic impedance, etc., can be computed accordingly. The components of the net reaction force acting on cylinder $i$ per unit length can be written in a matrix form [9] as

$$
\left.\left|F_{l}\right|=\left[\gamma_{j}^{A}\right]\left|\tilde{w}_{l}\right|+w\left[\gamma_{j}^{\prime}\right] \mid \dot{w}_{l}\right\}
$$

where $\left.\mid \bar{w}_{l}\right\}$ and $\left|\dot{w}_{l}\right|$ are, respectively, the column matrices

${ }^{3}$ In the combination of $i w_{1} i$ is defined as $\sqrt{ }-t$. of the acceleration and velocity of the oscillatine cylinder, $j$ and $l$ are from 1 to $2 K$. Matrix $[\gamma f]$ is called added-mass matrix and $\left[\gamma_{j i}^{\prime}\right]$ is called acoustic radiation-damping matrix, since the effect of the first term in equatiop (5) is to increase the inertias of the vibrating cylinders and the second term is to diminish the vibration amplizudes of the cylinders. The square matrices are proved symmetrical and dependent on the wavenumber, the cylinder l:adius, and the distance and orientation belween cylinders [9].

Numerical results for the added-mass coefficients and the acoustic radiation damping coefficients for twio cylinders of different radji in a row and for three cylinders and seven cylinders of equal radii arranqed in arrays are worked out and shown in Figs. 2 to 5.

The results piotted in Figs. 2 and 3 show for small dimensionless wavenumbers both the added-mass and acoustic radiation-damping coefficients are monotonically decreasing when the gap-to-radius ratin increases, and eventually the self added-mass coefficients approach unity and the mutual added-mass coefficients and the acoustical radiation-damping coefficients become zero as the gap-toradius ratio becomes sufficiently large $(G / K>0(1))$. The trend of the variation of these coefficients with the change of the gap-10-radias ratio is consistent with the results cbtained from incompressible flow thecry [10] and experiments [11]. For moderate and large dimensionless wavenuniber. the variation of these force coefficients with respect to a change of gap-to-radius ratio is noll simply monotonic because complicated interference effect:! (or coupling elfects) between waves and cylinders occur.

For a fixed value of gap-to-radius ratio, the variation of the acoustic reaction force coefficients with respect to a change of dimensionless wavenumbers can be seen from Figs. 4 and $S$. For infinitesimal dimensionless wavenumbers ( $k R \leq 0.03$ ), the added-mass coefficients art close to those obtained by incempressible flow theory [10], and the acoustic radiationdamping coefficients are practically zero. This implies that if the radius of the cylinder is very small compared to the acoustic wavelength, which is typical in many practical cas:<.

\section{Nomenclatuse}

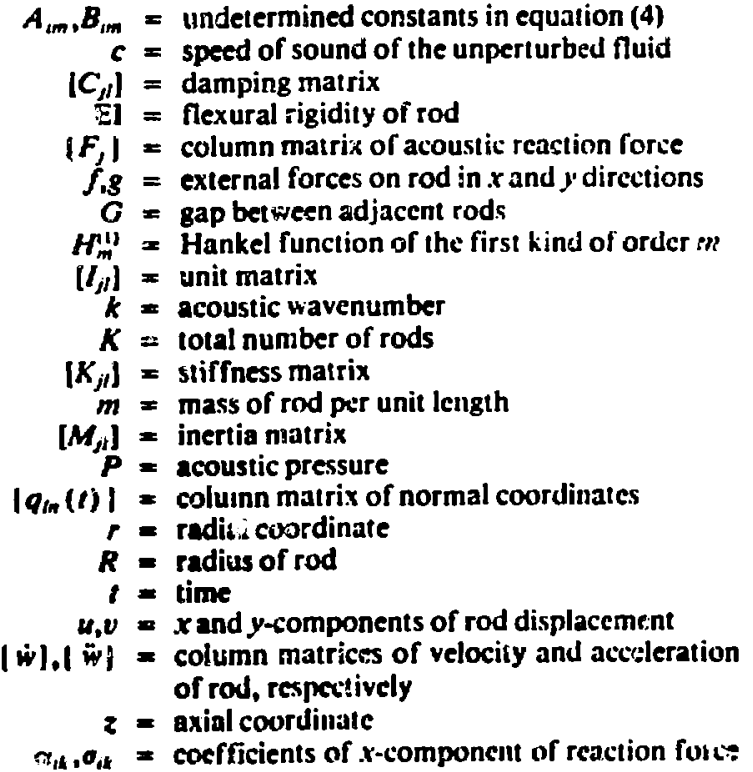

on rod $i$ due to the acceleration of $\operatorname{rod} k$ in $x$ and $y$ directions, respectively

$\beta_{i k}, \tau_{i k}=$ coefficients of $\left.y-c_{i}\right)$ mponent of reaction force on rod $i$ due to accileration of $\operatorname{rod} k$ in $x$ and $y$ directions, respect ivcly

$\eta(f), E(f)=$ normal coordinates

$\left[\gamma_{j}^{\prime}\right]=$ acoustic radiation-damping matrix

$\left[\gamma_{f}^{R}\right]=$ added-mass matrix

$\theta=$ angular coordinate

$\rho_{\infty}=$ density of unperturbed nuid

$\phi=$ velocity potential of perturbed nuid

$\omega=$ circular frequency of oscillation

$\zeta(z)=$ normal mode of rod in vacuo

$\nabla^{2}$ = Laplacian operator

Sobscripts

$i, k=$ refer to rod number, i.c., $1,2, \ldots, k$

$j, l=$ are from 1 to $2 K$

$m=$ refers to radial mode of acoustic wave

$n=$ refers to axial mode of nexural wave in rod

superscripts

$l=$ imaginary pari of complex number

$\boldsymbol{R}=$ real pari of complex number 

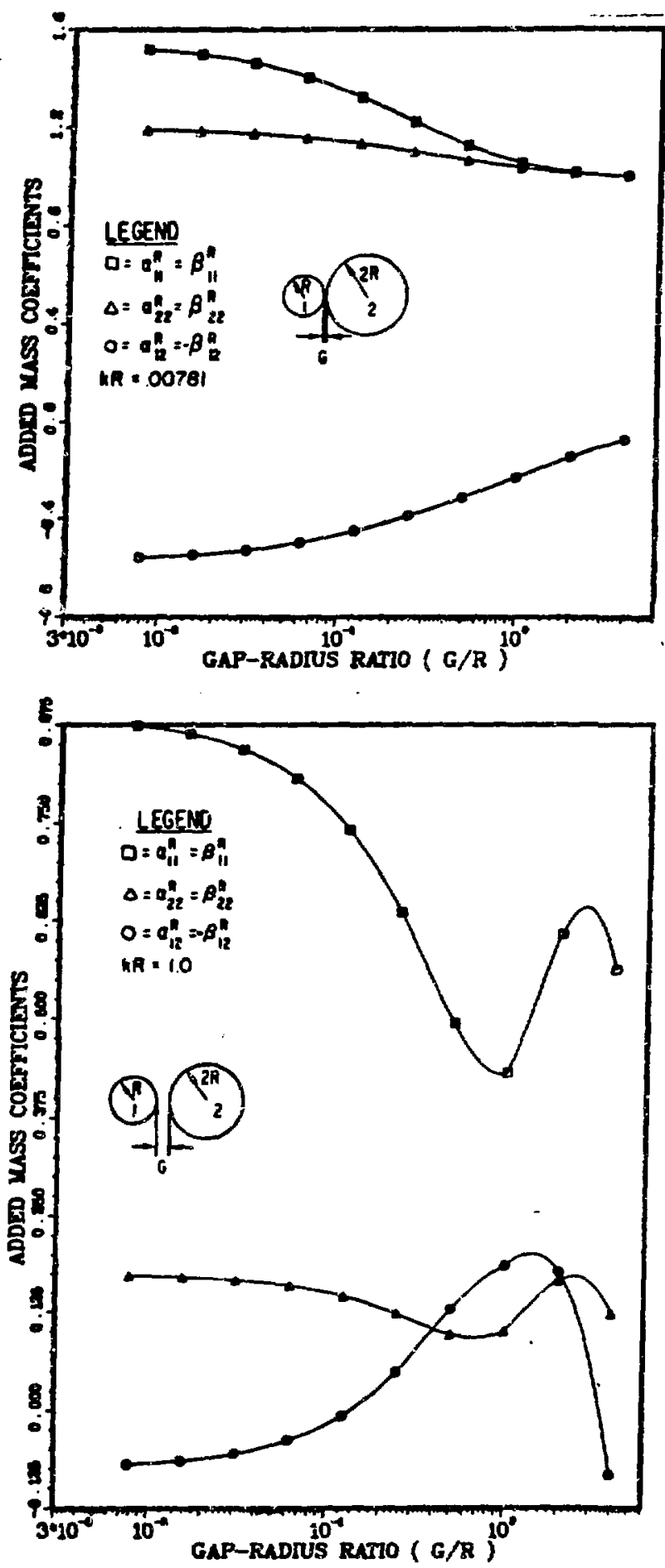

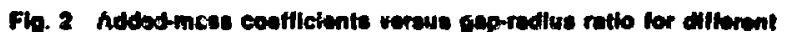
Animstontese wevenumbars

the effect of the added mass is dominant and the values of the added mass obtained by the incompressible potential theory can be used as valid approximation. As the dimensioniess wavenumber increases, the absolute values of boih the added mass and scoustic radiation-damping coefficicrists increase, reach to their maxima, and then decrease rapidly when the dimensionless wavenumber is increased further. When $k R \geq$ 1, these coefficients oscillate with respect to changes in the
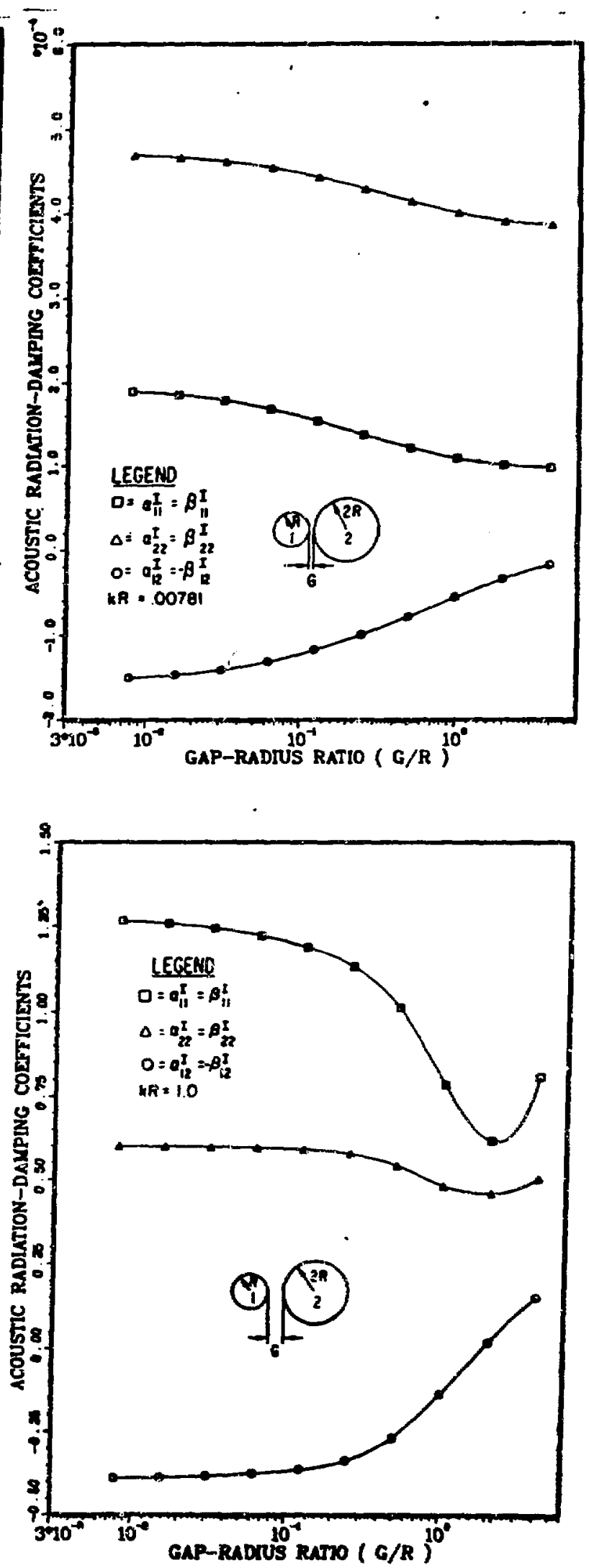

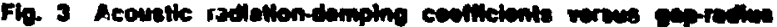

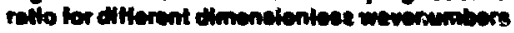



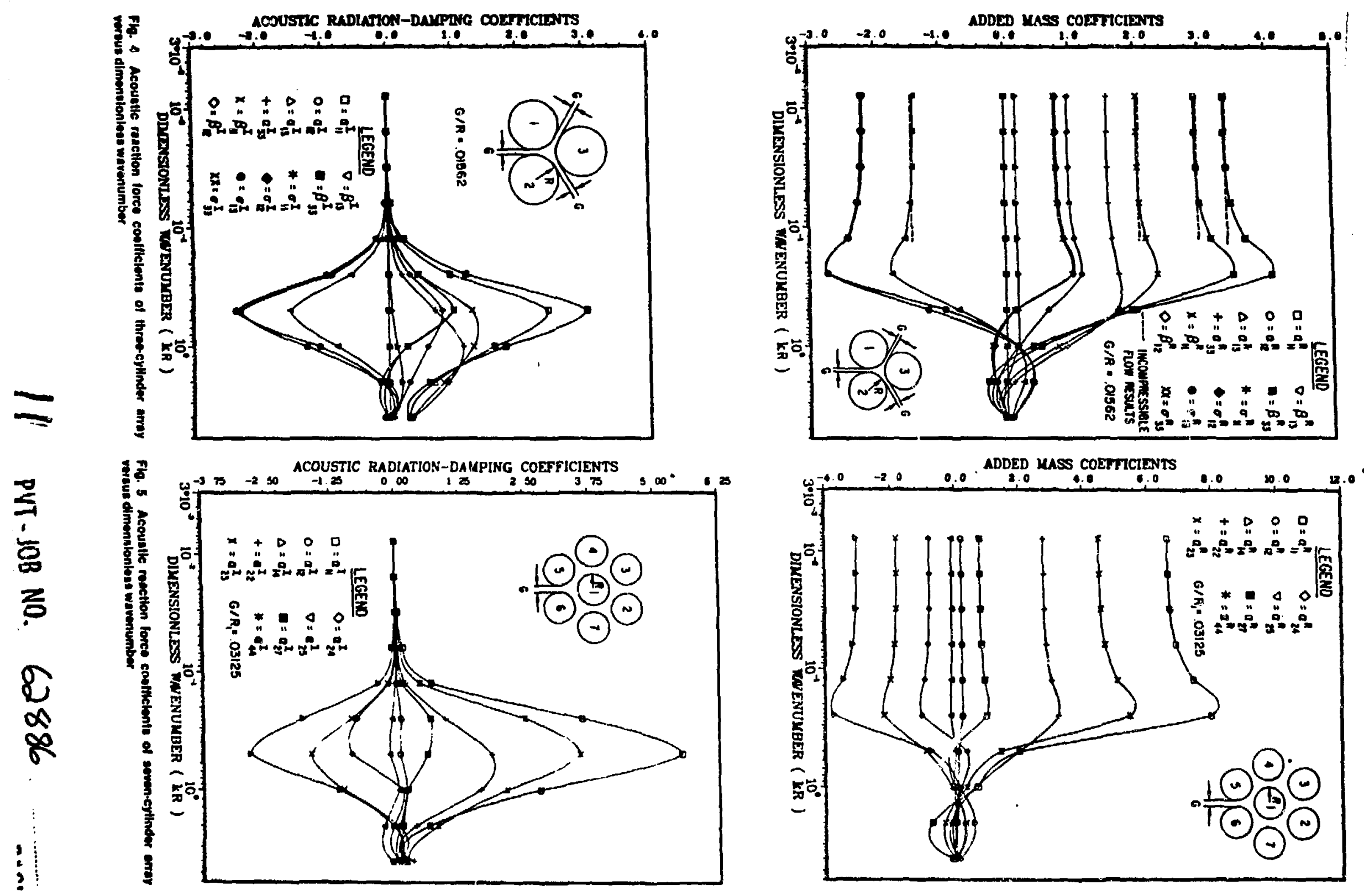
dimensionless wavenumber, and their absolute values are rencrally small.

\section{Vibration of a Cluster of Circulas Rods in on Infinite Acoustic Mediudn}

The acoustic reaction force derived in the previous section is used here as a forcing function for the vibration of a group of rods in an infinile acoustic medium. As shown in Fig. 1, each rod can move in the $x$ and $y$ directions. The equations of motion for rod $i$ are

$$
E_{i} I_{i} \frac{\partial^{4} u_{i}}{\partial z^{4}}+m_{i} \frac{\partial^{2} u_{i}}{\partial t^{2}}=f_{i}
$$

in the $x$ direction, and

$$
E_{i} I_{i} \frac{\partial^{4} v_{i}}{\partial z^{4}}+m_{i} \frac{\partial^{2} v_{i}}{\partial t^{2}}=g_{i}
$$

in the $y$ direction, where $E_{i} I_{l}$ is the flexural rigidity, $m_{i}$ the mass par unit leigth, $u_{i}, v_{i}$ the transverse displacements of rod $i_{1}$ and $f_{i}$ and $g_{1}$, respectively, the external forces on the rod in the $x$ and $y$ directions. If no other forces are involved in the acousto-elastic system, the force on the rod will be only the hydrodynamic reaction of the acoustic mediun.

Assuming all rods have the same types of boundary conditions in the $x$ and $y$ directions, then the normal modal functions in both directions will be the same. Using modal analysis, $u_{i}(z, \eta)$ and $v_{i}(z, i)$ can be expressed as

$$
\begin{aligned}
& u_{i}(z, n)=\sum_{n=1}^{\infty} \xi_{i n}(t) \zeta_{n}(z) \\
& v_{i}(z, t)=\sum_{n=1}^{\infty} \eta_{\text {in }}(t) \zeta_{n}(z)
\end{aligned}
$$

where $\xi_{m}(t)$ and $\eta_{i n}(t)$ are the normal condinates, and $\zeta_{n}(z)$ the normal mode of rods in vacuo. Substituting ex. pressions $(8,9)$ into equations $(6,7)$ and using expression (5) for the acoustic reaction force, multiplying the resulis by $\zeta_{m}(z)$, and iniegrating over the range of the span of a rod, we have the following set of orclirary equations in $\xi_{i r}(t)$ and $\eta_{n n}(t)$

$$
\left\{M, l\left|\ddot{q}_{i n}\right|+\left|C_{J}\right|\left|\dot{q}_{n n}\right|+\mid K_{j}\right]\left|a_{l n}\right|=|0|
$$

where

$$
\begin{aligned}
& \left.\left[M_{j,}\right]=\mid \gamma_{j}\right] \mid+m_{j}\left[l_{j}\right], j \text { and } l=1,2, \ldots, 2 K \text {, } \\
& \left.\left.\mid C_{j d}\right\rfloor=\left\lfloor\gamma_{j}^{\prime}\right\rfloor\right\rfloor \\
& {\left[K_{j}\right]=m_{j} \omega_{j n}^{2}\left[\boldsymbol{t}_{j}\right],} \\
& \left.\mid q_{i n}(i)\right)=\left\{\begin{array}{l}
z_{i n}(1) \\
\eta_{\text {in }}(1)
\end{array}\right\}, i=1,2, \ldots, K .
\end{aligned}
$$

$\left[I_{f l}\right]$ is a unit matrix,

and

$$
n=1,2,3, \ldots, \infty \text {. }
$$

Due ic acoustic coupling, $\xi_{\text {in }}(t)$ is coupled with $\eta_{\text {in }}(t)$ through the inertia and damping terms for rods oscillating in a stationary acoustic medium. For each value of $n$, there are $2 K$ equations in $\xi_{1 n}(t)$ and $\eta_{i n}(t)$. Equation $(I 0)$ is the basic equation used to study dyminics of the self-excited acoustoelastic system of a single rod or bundles of rods.

For harmonic motion of rods, we can write

$$
\boldsymbol{q}_{l n}=\hat{q}_{\ln } e^{-i \mathrm{w}}
$$

$\hat{q}_{1 n}$ being complex constants indicating the vibrational am- plitudes and phases. Substituting expression equation (10) and manipulating the resu't, we have

$$
\left.\left.\left\{-\left[M_{j}\right] \omega^{2}-\left[C_{j}\right] i \omega+\left\{K_{j}\right]\right\} \mid \dot{q}_{i n}\right\}=\mid 0\right\}
$$

The only nontrivial solutions for $\dot{g}_{\text {In }}$ are obtained under the condition that the determinant of the coefficients of equation (13) vanishes. This condition then leads to an eigenvalue cquation (or frequency equation) for the acousto-elastic sysiem, from which the eigen frequencies $\omega_{n}$ can be obtained.

Since the acoustic reaction force coefficients are dependent on wavenumber $\omega_{n} / c$, the frequency equation is highly Iranscendental. An iteration scheme is, therefore, needed to deterinine $\omega_{\text {n }}$ when the germetric arrangement of rod is known. Once the admissible frequency is obtained, the ratios of $\hat{q}_{l n} / \hat{q}_{I_{n}}$ can be determined from equation (13) and hence can the corresponding morle be obtzined.

Instead of solving the transcendental equations for frequencies and modes, the following example is presented to illustrate the effect of acoustic loading on the system parameters of the vibration of circular rods.

The speeds of sound in the air and in the water at room temiperalure are, respectively, about $110 \mathrm{ft} / \mathrm{s}$ and $4877 \mathrm{ft} / \mathrm{s}$. The radii $R$ of the fuel rods in a reactor arc of the order of 0.5 in. For low frequency vibration, say $f<200 \mathrm{~Hz}$, the values of dimensionless wavenumber $k R$ are 0.0476 and 0.0108 for rod/air and rod/water interactions. As shown in the previous section, the acoustic reaction force is independent of oscillation frequency and its real part (i.e., the acided mass) is more dorninant than its imaginary pert (i.e., the radiation damping) for $k R \leq \mathbf{0 . 0 3}$. The efrect of acoustic loading in this case is to reduce the values of natural frequencies of the coupled system. The coefficient matrix of equation (13) can te diagortalized to obtain natural frequencies and norma! modes of the system sizec $\left[C_{j l}\right]$, is vanishingly small for infinitesimal dimensionless wavenumbers.

For high frequency vibration, say $f>10^{4} \mid 12$, the values of $k k$ are 4.76 and 1.08 for rod/air and rod/water interactions, respectively. Both the added mass and the acoustic radiation damping are very smali for dimensionless wavenumbers grcater than unity. However, the acoustic radiation damping is more dominant than the added mass because a smal? accession in mass will not significantly alter the matuial frequencies while a small value of damping will cause energy to he ractiated. The effect of acousticat loading in this case is to change the total damping of the sysicm. The coupled frequencios of the system are approximalely the same; as in vacuo, but the corresponding modal shapes are altered by acoustic radiation damping.

For vibration of moderate frequencies such that the dinensionless wavenumber $k R$ being of order of unity, the interaction between the motion of the rods and the nujd becomes very complicated. The coupled frequencies and modal configurations must be determined by solving the transcendental equations. The acuustic loading alters both the frequencies and modal shapes of the acousto-structure system.

\section{Coasclusions}

This paper provides a general method of analysis for acoustic loading effect on circular rods oscillating in an infinite compressible fluid medium. The hydrodynamic reaction force due to the acoustic field generated by the moving rods is first solved, and the interaction between the motion of the rods and the fluid is then presented.

When the acoustic wavelength is large compared with the cylinder radius (i.e., $k R<<0$ (1), the added mass is more dominant than the radiation damping, and both are independent of oscillation frequency. Incompressible potential flow theory thus provides good approximation for the nuid

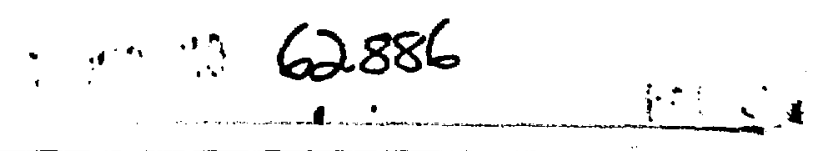


toading for low-frequency vibration. On the other hand, when the acoustic wavelength is small compared with the cylinder radius (i.e.. $\because R>O(1)$ ), the radiation damping is more dominant than the added mass, and both are small.

Fo: low-frequency oscillation, the effect of the acoustic loading is to alier the vibration characteristics, especially to reduce the natural frequency of the acousto-elastic system. For high-frequency oscillation, the acoustic loading does not change the coupled frequency, but the modal shape of the system.

\section{Acknowledgment}

This wor: was performed under the sponsorship of the Division of Reactor Research and Technology, U.S. Department of Energy.

\section{References}

I Faran, J. J., Jr., "Sound Scattering by Solid Cylinders and Spheres." Journat of ine Acoustical Society of Amerion. Yol. 23, No. 4, July 1951, pp. 405-418.
2 Junger, M.C., "Sound Scattering by Thin Elastic Shells," Journal of the Acoustical Society of America, Vol. 24, Nin. 4, July 1952, pp. 366-373.

3 Bajley, J. R., and Fahy, F. J. "Radiation and Response of Cylindrical Beams Exciled by Sound," ASAIE Jowrnal of Eingineering for Industry, June 1971. Paper No 71-Vibr-B4.

5 Hine, M. J., "Acoustically Induced Vibrations of Skender Rods in Cylindrical Duct." ASME Soursal of Apptied Mfechanics, Paper No. 72WA/APM+8,

5 Lin, H. C., sad Chen, 5. S., "Accustically Induced Vibration of Circulas Cylindrical Rods," Journal of Sownd ond Vibration, Vot. 31. No. 1. 1977, pp. 89-96.

6 Chen, S. S., "Vibrations of a Row of Circular Cylinders in a Liquid," ASME Journal of Endinering for Industry. Vol. 97, 1975, pp. 1212-1218.

7 Morse. P. M., and Ingard, K. U., Theorerical Acoustics, 2nd Edition. McGraw-Hill, New York, 1968.

8 Watson, G. N., Theory of Besset Functions, Cambridge Press, Cambridge, 1944.

9 Lin, W. K.. and Chen, S. S. "Acoustic Radiation and Scattering from a Growp of Circular Cylinders," ASHE Journal of Applied Mechonics, sub. mitted for publication.

10 Chen, S. S., "Dynamics of Heat Exohanger Tubr Banks," ASME Journal of Fluids Engineering. Vol. 99, 1977, pp. 462-469.

11 Moreti, P. M., and L.owcry, R. L.. "Hydrodynamic !nertia Coeficients for a Tube Surrounded by Rigid Tubes," ASME Jounal of l'Ressure Vesset TEChNOLOF, Vol. 94, 1976. pp. 190-193.

\section{DISCLAIMER}

This report was prepared as an account of work sponsored by an agency of the Uniled States Government. Neither the United States Government nor any agency thereof, nor any of their employees, makes any warranty, express or implied, or assumes any legal liatility or responsibility for the ascuracy, completencss, or usefulness of any information, apparatus, product, or process disclosed, or represents that its use would not infringe privately owned rights. Reference herein to any specific commercial product, process, or service by trade name, trademark, manufacturer, or otherwise does not necessarily constitute or imply its endorsement, recommendation, or favoring by the United States Government or any agency thereof. The vews and opinions of authors expresced herein do not necessarily state or reflect those of the United States Governinent or any agency thereof. 